\title{
Advanced nucleon EM structure model and its predictability
}

\author{
S.Dubnička*i \\ Institute of Physics, Slovak Academy of Sciences, Bratislava, Slovak Republic \\ E-mail: fyzidubnesavba.sk

\section{C.Adamuščín} \\ Institute of Physics, Slovak Academy of Sciences, Bratislava, Slovak Republic \\ E-mail: adamuscinesavba.sk

\section{E. Bartoš} \\ Institute of Physics, Slovak Academy of Sciences, Bratislava, Slovak Republic \\ E-mail: bartos@savba.sk
}

\section{A.Z. Dubničkova}

Department of Theoretical Physics, Comenius University, Bratislava, Slovak Republic

E-mail: dubnickova@fmph. uniba.sk

\begin{abstract}
The advanced 9 resonance nucleon electromagnetic structure model in the language of the isoscalar and iso-vector parts of the Dirac and Pauli form factors is demonstrated to describe all known experimental information. By means of such model the true behavior of the vector and tensor polarizations in $e^{+} e^{-} \rightarrow p \bar{p}$ process are predicted. The model, in combinations with $S U$ (3) symmetry, is able to predict behaviors of electromagnetic form factors of all $1 / 2^{+}$octet hyperons as well.
\end{abstract}

The European Physical Society Conference on High Energy Physics -EPS-HEP2013

18-24 July 2013

Stockholm, Sweden

* Speaker.

${ }^{\dagger}$ A footnote may follow. 


\section{Introduction}

The experimental data on the nucleon electromagnetic (EM) structure are presented in the language of the electric and magnetic form factors (FFs) of proton and neutron $G_{E}^{p}(t) G_{M}^{p}(t), G_{E}^{n}(t)$, $G_{M}^{n}(t)$, respectively.

However, for construction of various nucleon EM structure models the flavor-independent isoscalar and iso-vector parts of the Dirac and Pauli FFs are more suitable.

Both sets are connected by the relatios

$G_{E}^{p}(t)=\left[F_{1 N}^{s}(t)+F_{1 N}^{v}(t)\right]+\frac{t}{4 m_{p}^{2}}\left[F_{2 N}^{s}(t)+F_{2 N}^{v}(t)\right]$

$G_{M}^{p}(t)=\left[F_{1 N}^{s}(t)+F_{1 N}^{v}(t)\right]+\left[F_{2 N}^{s}(t)+F_{2 N}^{v}(t)\right]$

$G_{E}^{n}(t)=\left[F_{1 N}^{s}(t)-F_{1 N}^{v}(t)\right]+\frac{t}{4 m_{n}^{2 N}}\left[F_{2 N}^{s}(t)-F_{2 N}^{v}(t)\right]$

$G_{M}^{n}(t)=\left[F_{1 N}^{s}(t)-F_{1 N}^{v}(t)\right]+\left[F_{2 N}^{s}(t)-F_{2 N}^{v}(t)\right]$

\section{Advanced model of nucleon EM structure}

In this contribution we would like to present 9 resonance Unitary\&Analytic (U\&A) model respecting $S U(3)$ classification of hadrons, including also the OZI rule violation.

Consideration of the $S U(3)$ symmetry means that always complete trinity of vector-mesons $\left(\rho, \omega, \phi ; \rho^{\prime}, \omega^{\prime}, \phi^{\prime} ;\right.$ etc. $)$ has to be taken into account.

The newest Review of Particle Physics [1] provides just 3 complete trinities of such vectormeson resonances

$\rho(770), \omega(782), \phi(1020)$

$\left.\omega^{\prime}(1420), \rho^{\prime}(1450)\right), \phi^{\prime}(1680)$

$\omega^{\prime \prime}(1650), \rho^{\prime \prime}(1700), \phi^{\prime \prime}(2170)$

then also OZI rule violation is fulfilled as the nonzero contributions of the $\phi$ mesons are considered.

As a result the 9-resonance (3 iso-vectors and 6 iso-scalars) zero-width $V M D$ parametrization of $F_{1 N}^{s}(t), F_{1 N}^{v}(t), F_{2 N}^{s}(t), F_{2 N}^{v}(t)$ can be written down

$$
\begin{array}{r}
F_{1 N}^{S}(t)=\frac{1}{2} \frac{m_{\omega}^{2} m_{\phi}^{2}}{\left(m_{\omega}^{2}-t\right)\left(m_{\phi}^{2}-t\right)}+ \\
+\left\{\frac{m_{\phi}^{2} m_{\omega^{\prime}}^{2}}{\left(m_{\phi}^{2}-t\right)\left(m_{\omega^{\prime}}^{2}-t\right)} \frac{\left(m_{\phi}^{2}-m_{\omega^{\prime}}^{2}\right)}{\left(m_{\phi}^{2}-m_{\omega}^{2}\right)}+\frac{m_{\omega}^{2} m_{\omega^{\prime}}^{2}}{\left(m_{\omega}^{2}-t\right)\left(m_{\omega^{\prime}}^{2}-t\right)} \frac{\left(m_{\omega}^{2}-m_{\omega^{\prime}}^{2}\right)}{\left(m_{\omega}^{2}-m_{\phi}^{2}\right)}-\right. \\
\left.+\frac{m_{\omega}^{2} m_{\phi}^{2}}{\left(m_{\omega}^{2}-t\right)\left(m_{\phi}^{2}-t\right)}\right\}\left(f_{\omega^{\prime} N N}^{(1)} / f_{\omega^{\prime}}\right)+ \\
+\left\{\frac{m_{\phi}^{2} m_{\phi^{\prime}}^{2}}{\left(m_{\phi}^{2}-t\right)\left(m_{\phi^{\prime}}^{2}-t\right)} \frac{\left(m_{\phi}^{2}-m_{\phi^{\prime}}^{2}\right)}{\left(m_{\phi}^{2}-m_{\omega}^{2}\right)}+\frac{m_{\omega}^{2} m_{\phi^{\prime}}^{2}}{\left(m_{\omega}^{2}-t\right)\left(m_{\phi^{\prime}}^{2}-t\right)} \frac{\left(m_{\omega}^{2}-m_{\phi^{\prime}}^{2}\right)}{\left(m_{\omega}^{2}-m_{\phi}^{2}\right)}-\right. \\
+\left\{\frac{m_{\omega}^{2} m_{\phi}^{2}}{\left(m_{\omega}^{2}-t\right)\left(m_{\phi}^{2}-t\right)}\right\}\left(f_{\phi^{\prime} N N}^{(1)} / f_{\phi^{\prime}}\right)+ \\
\left(m_{\phi}^{2}-t\right)\left(m_{\omega^{\prime \prime}}^{2}-t\right)
\end{array}
$$




$$
\begin{aligned}
& \left.-\frac{m_{\omega}^{2} m_{\phi}^{2}}{\left(m_{\omega}^{2}-t\right)\left(m_{\phi}^{2}-t\right)}\right\}\left(f_{\omega^{\prime \prime} N N}^{(1)} / f_{\omega^{\prime \prime}}\right)+ \\
& +\left\{\frac{m_{\phi}^{2} m_{\phi^{\prime \prime}}^{2}}{\left(m_{\phi}^{2}-t\right)\left(m_{\phi^{\prime \prime}}^{2}-t\right)} \frac{\left(m_{\phi}^{2}-m_{\phi^{\prime \prime}}^{2}\right)}{\left(m_{\phi}^{2}-m_{\omega}^{2}\right)}+\frac{m_{\omega}^{2} m_{\phi^{\prime \prime}}^{2}}{\left(m_{\omega}^{2}-t\right)\left(m_{\phi^{\prime \prime}}^{2}-t\right)} \frac{\left(m_{\omega}^{2}-m_{\phi^{\prime \prime}}^{2}\right)}{\left(m_{\omega}^{2}-m_{\phi}^{2}\right)}-\right. \\
& \left.-\frac{m_{\omega}^{2} m_{\phi}^{2}}{\left(m_{\omega}^{2}-t\right)\left(m_{\phi}^{2}-t\right)}\right\}\left(f_{\phi^{\prime \prime} N N}^{(1)} / f_{\phi^{\prime \prime}}\right) \text {. } \\
& F_{1 N}^{v}(t)=\frac{1}{2} \frac{m_{\rho}^{2} m_{\rho^{\prime}}^{2}}{\left(m_{\rho}^{2}-t\right)\left(m_{\rho^{\prime}}^{2}-t\right)}+ \\
& +\left\{\frac{m_{\rho^{\prime}}^{2} m_{\rho^{\prime \prime}}^{2}}{\left(m_{\rho^{\prime}}^{2}-t\right)\left(m_{\rho^{\prime \prime}}^{2}-t\right)} \frac{\left(m_{\rho^{\prime}}^{2}-m_{\rho^{\prime \prime}}^{2}\right)}{\left(m_{\rho^{\prime}}^{2}-m_{\rho}^{2}\right)}+\frac{m_{\rho}^{2} m_{\rho^{\prime \prime}}^{2}}{\left(m_{\rho}^{2}-t\right)\left(m_{\rho^{\prime \prime}}^{2}-t\right)} \frac{\left(m_{\rho}^{2}-m_{\rho^{\prime \prime}}^{2}\right)}{\left(m_{\rho}^{2}-m_{\rho^{\prime}}^{2}\right)}-\right. \\
& \left.-\frac{m_{\rho}^{2} m_{\rho^{\prime}}^{2}}{\left(m_{\rho}^{2}-t\right)\left(m_{\rho^{\prime}}^{2}-t\right)}\right\}\left(f_{\rho^{\prime \prime} N N}^{(1)} / f_{\rho^{\prime \prime}}\right) \text {. } \\
& F_{2 N}^{s}(t)=\frac{1}{2}\left(\mu_{p}+\mu_{n}\right) \frac{m_{\omega}^{2} m_{\phi}^{2} m_{\omega^{\prime}}^{2}}{\left(m_{\omega}^{2}-t\right)\left(m_{\phi}^{2}-t\right)\left(m_{\omega^{\prime}}^{2}-t\right)}+ \\
& +\left\{\frac{m_{\phi}^{2} m_{\omega^{\prime}}^{2} m_{\phi^{\prime}}^{2}}{\left(m_{\phi}^{2}-t\right)\left(m_{\omega^{\prime}}^{2}-t\right)\left(m_{\phi^{\prime}}^{2}-t\right)} \frac{\left(m_{\phi}^{2}-m_{\phi^{\prime}}^{2}\right)\left(m_{\omega^{\prime}}^{2}-m_{\phi^{\prime}}^{2}\right)}{\left(m_{\phi}^{2}-m_{\omega}^{2}\right)\left(m_{\omega^{\prime}}^{2}-m_{\omega}^{2}\right)}+\right. \\
& +\frac{m_{\omega}^{2} m_{\omega^{\prime}}^{2} m_{\phi^{\prime}}^{2}}{\left(m_{\omega}^{2}-t\right)\left(m_{\omega^{\prime}}^{2}-t\right)\left(m_{\phi^{\prime}}^{2}-t\right)} \frac{\left(m_{\omega}^{2}-m_{\phi^{\prime}}^{2}\right)\left(m_{\omega^{\prime}}^{2}-m_{\phi^{\prime}}^{2}\right)}{\left(m_{\omega}^{2}-m_{\phi}^{2}\right)\left(m_{\omega^{\prime}}^{2}-m_{\phi}^{2}\right)}+ \\
& +\frac{m_{\omega}^{2} m_{\phi^{2}}^{2} m_{\phi^{\prime}}^{2}}{\left(m_{\omega}^{2}-t\right)\left(m_{\phi}^{2}-t\right)\left(m_{\phi^{\prime}}^{2}-t\right)} \frac{\left(m_{\omega}^{2}-m_{\phi^{\prime}}^{2}\right)\left(m_{\phi}^{2}-m_{\phi^{\prime}}^{2}\right)}{\left(m_{\omega}^{2}-m_{\omega^{\prime}}^{2}\right)\left(m_{\phi}^{2}-m_{\omega^{\prime}}^{2}\right)}- \\
& \left.-\frac{m_{\omega}^{2} m_{\phi}^{2} m_{\omega^{\prime}}^{2}}{\left(m_{\omega}^{2}-t\right)\left(m_{\phi}^{2}-t\right)\left(m_{\omega^{\prime}}^{2}-t\right)}\right\}\left(f_{\phi^{\prime} N N}^{(2)} / f_{\phi^{\prime}}\right)+ \\
& +\left\{\frac{m_{\phi}^{2} m_{\omega^{\prime}}^{2} m_{\omega^{\prime \prime}}^{2}}{\left(m_{\phi}^{2}-t\right)\left(m_{\omega^{\prime}}^{2}-t\right)\left(m_{\omega^{\prime \prime}}^{2}-t\right)} \frac{\left(m_{\phi}^{2}-m_{\omega^{\prime \prime}}^{2}\right)\left(m_{\omega^{\prime}}^{2}-m_{\omega^{\prime \prime}}^{2}\right)}{\left(m_{\phi}^{2}-m_{\omega}^{2}\right)\left(m_{\omega^{\prime}}^{2}-m_{\omega}^{2}\right)}+\right. \\
& +\frac{m_{\omega}^{2} m_{\omega^{\prime}}^{2} m_{\omega^{\prime \prime}}^{2}}{\left(m_{\omega}^{2}-t\right)\left(m_{\omega^{\prime}}^{2}-t\right)\left(m_{\omega^{\prime \prime}}^{2}-t\right)} \frac{\left(m_{\omega}^{2}-m_{\omega^{\prime \prime}}^{2}\right)\left(m_{\omega^{\prime}}^{2}-m_{\omega^{\prime \prime}}^{2}\right)}{\left(m_{\omega}^{2}-m_{\phi}^{2}\right)\left(m_{\omega^{\prime}}^{2}-m_{\phi}^{2}\right)}+ \\
& +\frac{m_{\omega^{\prime}}^{2} m_{\phi}^{2} m_{\omega^{\prime \prime}}^{2}}{\left(m_{\omega}^{2}-t\right)\left(m_{\phi}^{2}-t\right)\left(m_{\omega^{\prime \prime}}^{2}-t\right)} \frac{\left(m_{\omega}^{2}-m_{\omega^{\prime \prime}}^{2}\right)\left(m_{\phi}^{2}-m_{\omega^{\prime \prime}}^{2}\right)}{\left(m_{\omega}^{2}-m_{\omega^{\prime}}^{2}\right)\left(m_{\phi}^{2}-m_{\omega^{\prime}}^{2}\right)}- \\
& \left.-\frac{m_{\omega}^{2} m_{\phi}^{2} m_{\omega^{\prime}}^{2}}{\left(m_{\omega}^{2}-t\right)\left(m_{\phi}^{2}-t\right)\left(m_{\omega^{\prime}}^{2}-t\right)}\right\}\left(f_{\omega^{\prime \prime} N N}^{(2)} / f_{\omega^{\prime \prime}}\right)+ \\
& +\left\{\frac{m_{\phi}^{2} m_{\omega^{\prime}}^{2} m_{\phi^{\prime \prime}}^{2}}{\left(m_{\phi}^{2}-t\right)\left(m_{\omega^{\prime}}^{2}-t\right)\left(m_{\phi^{\prime \prime}}^{2}-t\right)} \frac{\left(m_{\phi}^{2}-m_{\phi^{\prime \prime}}^{2}\right)\left(m_{\omega^{\prime}}^{2}-m_{\phi^{\prime \prime}}^{2}\right)}{\left(m_{\phi}^{2}-m_{\omega}^{2}\right)\left(m_{\omega^{\prime}}^{2}-m_{\omega}^{2}\right)}+\right. \\
& +\frac{m_{\omega}^{2} m_{\omega^{\prime}}^{2} m_{\phi^{\prime \prime}}^{2}}{\left(m_{\omega}^{2}-t\right)\left(m_{\omega^{\prime}}^{2}-t\right)\left(m_{\phi^{\prime \prime}}^{2}-t\right)} \frac{\left(m_{\omega}^{2}-m_{\phi^{\prime \prime}}^{2}\right)\left(m_{\omega^{\prime}}^{2}-m_{\phi^{\prime \prime}}^{2}\right)}{\left(m_{\omega}^{2}-m_{\phi}^{2}\right)\left(m_{\omega^{\prime}}^{2}-m_{\phi}^{2}\right)}+
\end{aligned}
$$




$$
\begin{gathered}
+\frac{m_{\omega}^{2} m_{\phi}^{2} m_{\phi^{\prime \prime}}^{2}}{\left(m_{\omega}^{2}-t\right)\left(m_{\phi}^{2}-t\right)\left(m_{\phi^{\prime \prime}}^{2}-t\right)} \frac{\left(m_{\omega}^{2}-m_{\phi^{\prime \prime}}^{2}\right)\left(m_{\phi}^{2}-m_{\phi^{\prime \prime}}^{2}\right)}{\left(m_{\omega}^{2}-m_{\omega^{\prime}}^{2}\right)\left(m_{\phi}^{2}-m_{\omega^{\prime}}^{2}\right)}- \\
\left.-\frac{m_{\omega^{2}}^{2} m_{\phi}^{2} m_{\omega^{\prime}}^{2}}{\left(m_{\omega}^{2}-t\right)\left(m_{\phi}^{2}-t\right)\left(m_{\omega^{\prime}}^{2}-t\right)}\right\}\left(f_{\phi^{\prime \prime} N N}^{(2)} / f_{\phi^{\prime \prime}}\right) \\
F_{2 N}^{v}(t)=\frac{1}{2} \frac{m_{\rho}^{2} m_{\rho^{\prime}}^{2} m_{\rho^{\prime \prime}}^{2}}{\left(m_{\rho}^{2}-t\right)\left(m_{\rho^{\prime}}^{2}-t\right)\left(m_{\rho^{\prime \prime}}^{2}-t\right)}
\end{gathered}
$$

to be automatically normalized and governing the asymptotic behaviors as predicted by the quark model of hadrons.

By the non-linear transformations

$t=t_{0}^{s}+\frac{4\left(t_{\text {in }}^{1 s}-t_{0}^{s}\right)}{[1 / V(t)-V(t)]^{2}} ; \quad t=t_{0}^{v}+\frac{4\left(t_{i n}^{1 v}-t_{0}^{v}\right)}{[1 / W(t)-W(t)]^{2}} ;$

$t=t_{0}^{s}+\frac{4\left(t_{i n}^{2 s}-t_{0}^{s}\right)}{[1 / U(t)-U(t)]^{2}} ; \quad t=t_{0}^{v}+\frac{4\left(t_{i n}^{2 v}-t_{0}^{v}\right)}{[1 / X(t)-X(t)]^{2}}$.

and a subsequent inclusion of the nonzero values of vector-meson widths, for every iso-scalar and iso-vector Dirac and Pauli FF, one obtains just one analytic and smooth from $-\infty$ to $+\infty$ function in the forms

$$
\begin{aligned}
& F_{1 N}^{s}[V(t)]=\left(\frac{1-V^{2}}{1-V_{N}^{2}}\right)^{4}\left\{\frac{1}{2} L_{\omega}(V) L_{\phi}(V)+\right. \\
& +\left[L_{\phi}(V) L_{\omega^{\prime}}(V) \frac{\left(C_{\phi}^{1 s}-C_{\omega^{\prime}}^{1 s}\right)}{\left(C_{\phi}^{1 s}-C_{\omega}^{1 s}\right)}+L_{\omega}(V) L_{\omega^{\prime}}(V) \frac{\left(C_{\omega}^{1 s}-C_{\omega^{\prime}}^{1 s}\right)}{\left(C_{\omega}^{1 s}-C_{\phi}^{1 s}\right)}-L_{\omega}(V) L_{\phi}(V)\right]\left(f_{\omega^{\prime} N N}^{(1)} / f_{\omega^{\prime}}\right)+ \\
& +\left[L_{\phi}(V) H_{\phi^{\prime}}(V) \frac{\left(C_{\phi}^{1 s}-C_{\phi^{\prime}}^{1 s}\right)}{\left(C_{\phi}^{1 s}-C_{\omega}^{1 s}\right)}+L_{\omega}(V) H_{\phi^{\prime}}(V) \frac{\left(C_{\omega}^{1 s}-C_{\phi^{\prime}}^{1 s}\right)}{\left(C_{\omega}^{1 s}-C_{\phi}^{1 s}\right)}-L_{\omega}(V) L_{\phi}(V)\right]\left(f_{\phi^{\prime} N N}^{(1)} / f_{\phi^{\prime}}\right)+ \\
& +\left[L_{\phi}(V) H_{\omega^{\prime \prime}}(V) \frac{\left(C_{\phi}^{1 s}-C_{\omega^{\prime \prime}}^{1 s}\right)}{\left(C_{\phi}^{1 s}-C_{\omega}^{1 s}\right)}+L_{\omega}(V) H_{\omega^{\prime \prime}}(V) \frac{\left(C_{\omega}^{1 s}-C_{\omega^{\prime \prime}}^{1 s}\right)}{\left(C_{\omega}^{1 s}-C_{\phi}^{1 s}\right)}-L_{\omega}(V) L_{\phi}(V)\right]\left(f_{\omega^{\prime \prime} N N}^{(1)} / f_{\omega^{\prime \prime}}\right)+ \\
& \left.+\left[L_{\phi}(V) H_{\phi^{\prime \prime}}(V) \frac{\left(C_{\phi}^{1 s}-C_{\phi^{\prime \prime}}^{1 s}\right)}{\left(C_{\phi}^{1 s}-C_{\omega}^{1 s}\right)}+L_{\omega}(V) H_{\phi^{\prime \prime}}(V) \frac{\left(C_{\omega}^{1 s}-C_{\phi^{\prime \prime}}^{1 s}\right)}{\left(C_{\omega}^{1 s}-C_{\phi}^{1 s}\right)}-L_{\omega}(V) L_{\phi}(V)\right]\left(f_{\phi^{\prime \prime} N N}^{(1)} / f_{\phi^{\prime \prime}}\right)\right\} \\
& F_{1 N}^{v}[W(t)]=\left(\frac{1-W^{2}}{1-W_{N}^{2}}\right)^{4}\left\{\frac{1}{2} L_{\rho}(W) L_{\rho^{\prime}}(W)+\right. \\
& \left.+\left[L_{\rho^{\prime}}(W) H_{\rho^{\prime \prime}}(W) \frac{\left(C_{\rho^{\prime}}^{1 v}-C_{\rho^{\prime \prime}}^{1 v}\right)}{\left(C_{\rho^{\prime}}^{1 v}-C_{\rho}^{1 v}\right)}+L_{\rho}(W) H_{\rho^{\prime \prime}}(W) \frac{\left(C_{\rho}^{1 v}-C_{\rho^{\prime \prime}}^{1 v}\right)}{\left(C_{\rho}^{1 v}-C_{\rho^{\prime}}^{1 v}\right)}-L_{\rho}(W) L_{\rho^{\prime}}(W)\right]\left(f_{\rho^{\prime \prime} N N}^{(1)} / f_{\rho^{\prime \prime}}\right)\right\} \\
& F_{2 N}^{s}[U(t)]=\left(\frac{1-U^{2}}{1-U_{N}^{2}}\right)^{6}\left\{\frac{1}{2}\left(\mu_{p}+\mu_{n}\right) L_{\omega}(U) L_{\phi}(U) L_{\omega^{\prime}}(U)+\right. \\
& +\left[L_{\phi}(U) L_{\omega^{\prime}}(U) H_{\phi^{\prime}}(U) \frac{\left(C_{\phi}^{2 s}-C_{\phi^{\prime}}^{2 s}\right)\left(C_{\omega^{\prime}}^{2 s}-C_{\phi^{\prime}}^{2 s}\right)}{\left(C_{\phi}^{2 s}-C_{\omega}^{2 s}\right)\left(C_{\omega^{\prime}}^{2 s}-C_{\omega}^{2 s}\right)}+\right.
\end{aligned}
$$




$$
\begin{aligned}
& +L_{\omega}(U) L_{\omega^{\prime}}(U) H_{\phi^{\prime}}(U) \frac{\left(C_{\omega}^{2 s}-C_{\phi^{\prime}}^{2 s}\right)\left(C_{\omega^{\prime}}^{2 s}-C_{\phi^{\prime}}^{2 s}\right)}{\left(C_{\omega}^{2 s}-C_{\phi}^{2 s}\right)\left(C_{\omega^{\prime}}^{2 s}-C_{\phi}^{2 s}\right)}+ \\
& +L_{\omega}(U) L_{\phi}(U) H_{\phi^{\prime}}(U) \frac{\left(C_{\omega}^{2 s}-C_{\phi^{\prime}}^{2 s}\right)\left(C_{\phi}^{2 s}-C_{\phi^{\prime}}^{2 s}\right)}{\left(C_{\omega}^{2 s}-C_{\omega^{\prime}}^{2 s}\right)\left(C_{\phi}^{2 s}-C_{\omega^{\prime}}^{2 s}\right)}- \\
& \left.-L_{\omega}(U) L_{\phi}(U) L_{\omega^{\prime}}(U)\right]\left(f_{\phi^{\prime} N N}^{(2)} / f_{\phi^{\prime}}\right)+ \\
& +\left[L_{\phi}(U) L_{\omega^{\prime}}(U) H_{\omega^{\prime \prime}}(U) \frac{\left(C_{\phi}^{2 s}-C_{\omega^{\prime \prime}}^{2 s}\right)\left(C_{\omega^{\prime}}^{2 s}-C_{\omega^{\prime \prime}}^{2 s}\right)}{\left(C_{\phi}^{2 s}-C_{\omega}^{2 s}\right)\left(C_{\omega^{\prime}}^{2 s}-C_{\omega}^{2 s}\right)}+\right. \\
& +L_{\omega}(U) L_{\omega^{\prime}}(U) H_{\omega^{\prime \prime}}(U) \frac{\left(C_{\omega}^{2 s}-C_{\omega^{\prime \prime}}^{2 s}\right)\left(C_{\omega^{\prime}}^{2 s}-C_{\omega^{\prime \prime}}^{2 s}\right)}{\left(C_{\omega}^{2 s}-C_{\phi}^{2 s}\right)\left(C_{\omega^{\prime}}^{2 s}-C_{\phi}^{2 s}\right)}+ \\
& +L_{\omega}(U) L_{\phi}(U) H_{\omega^{\prime \prime}}(U) \frac{\left(C_{\omega}^{2 s}-C_{\omega^{\prime \prime}}^{2 s}\right)\left(C_{\phi}^{2 s}-C_{\omega^{\prime \prime}}^{2 s}\right)}{\left(C_{\omega}^{2 s}-C_{\omega^{\prime}}^{2 s}\right)\left(C_{\phi}^{2 s}-C_{\omega^{\prime}}^{2 s}\right)}- \\
& \left.-L_{\omega}(U) L_{\phi}(U) L_{\omega^{\prime}}(U)\right]\left(f_{\omega^{\prime \prime} N N}^{(2)} / f_{\omega^{\prime \prime}}\right)+ \\
& +\left[L_{\phi}(U) L_{\omega^{\prime}}(U) H_{\phi^{\prime \prime}}(U) \frac{\left(C_{\phi}^{2 s}-C_{\phi^{\prime \prime}}^{2 s}\right)\left(C_{\omega^{\prime}}^{2 s}-C_{\phi^{\prime \prime}}^{2 s}\right)}{\left(C_{\phi}^{2 s}-C_{\omega}^{2 s}\right)\left(C_{\omega^{\prime}}^{2 s}-C_{\omega}^{2 s}\right)}+\right. \\
& +L_{\omega}(U) L_{\omega^{\prime}}(U) H_{\phi^{\prime \prime}}(U) \frac{\left(C_{\omega}^{2 s}-C_{\phi^{\prime \prime}}^{2 s}\right)\left(C_{\omega^{\prime}}^{2 s}-C_{\phi^{\prime \prime}}^{2 s}\right)}{\left(C_{\omega}^{2 s}-C_{\phi}^{2 s}\right)\left(C_{\omega^{\prime}}^{2 s}-C_{\phi}^{2 s}\right)}+ \\
& +L_{\omega}(U) L_{\phi}(U) H_{\phi^{\prime \prime}}(U) \frac{\left(C_{\omega}^{2 s}-C_{\phi^{\prime \prime}}^{2 s}\right)\left(C_{\phi}^{2 s}-C_{\phi^{\prime \prime}}^{2 s}\right)}{\left(C_{\omega}^{2 s}-C_{\omega^{\prime}}^{2 s}\right)\left(C_{\phi}^{2 s}-C_{\omega^{\prime}}^{2 s}\right)}- \\
& \left.\left.-L_{\omega}(U) L_{\phi}(U) L_{\omega^{\prime}}(U)\right]\left(f_{\phi^{\prime \prime N N}}^{(2)} / f_{\phi^{\prime \prime}}\right)\right\} \\
& F_{2 N}^{v}[X(t)]=\left(\frac{1-X^{2}}{1-X_{N}^{2}}\right)^{6}\left\{\frac{1}{2}\left(\mu_{p}-\mu_{n}\right) L_{\rho}(U) L_{\rho^{\prime}}(U) H_{\rho^{\prime \prime}}(U)\right\}
\end{aligned}
$$

with denotations from ref.[2].

This advanced model is defined on four-sheeted Riemann surface and includes all required nucleon FFs properties. Moreover, the model describes all today's nucleon EM FFs data in spacelike and time-like regions simultaneously as it is presented in Figs. 1-3.

\section{Vector and tensor polarizations in $e^{+} e^{-} \rightarrow p \bar{p}$ process}

The advanced $U \& A$ nucleon EM structure model is analytic and so it creates the nonzero imaginary parts of the FFs starting from the lowest branch points on the positive real axis in a natural way. Therefore, it can be applied for the predictions [3] of the vector

$$
P_{x}=-\frac{2 \sin \theta \cdot \operatorname{Re}\left[G_{E p}(s) G_{M p}^{*}(s)\right] \tau}{\left|G_{E p}(s)\right|^{2} \sin ^{2} \theta / \tau+\left|G_{M p}(s)\right|^{2}\left(1+\cos ^{2} \theta\right)}
$$



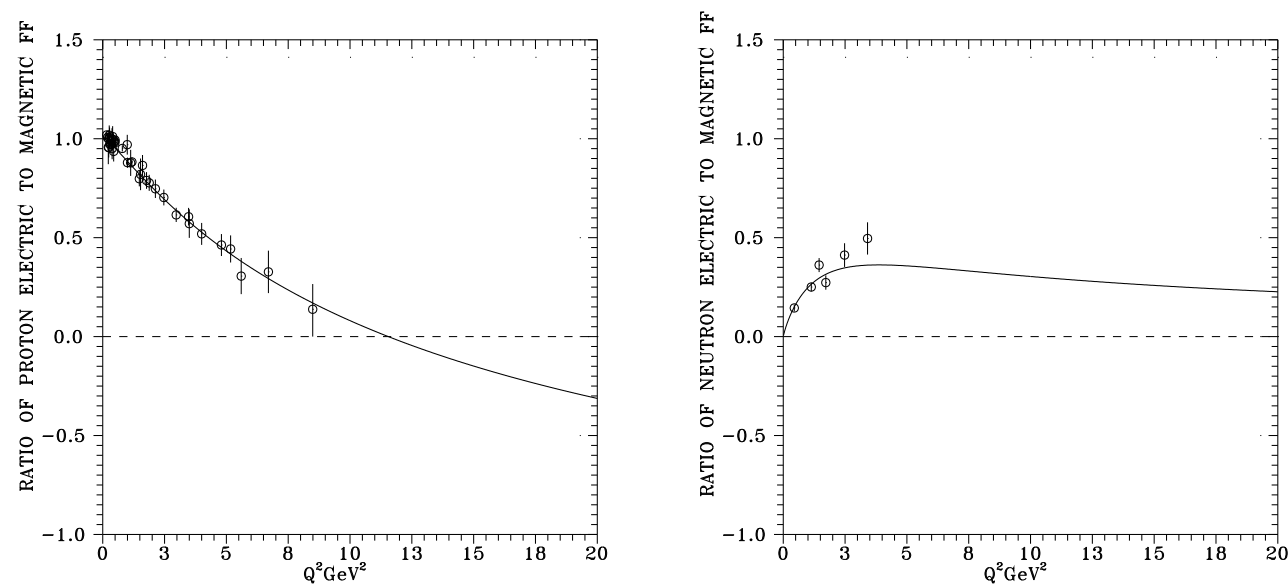

Figure 1: Prediction of proton and neutron electric to magnetic FFs ratio behavior by $U \& A$ model respecting $S U(3)$ symmetry and OZI rule violation
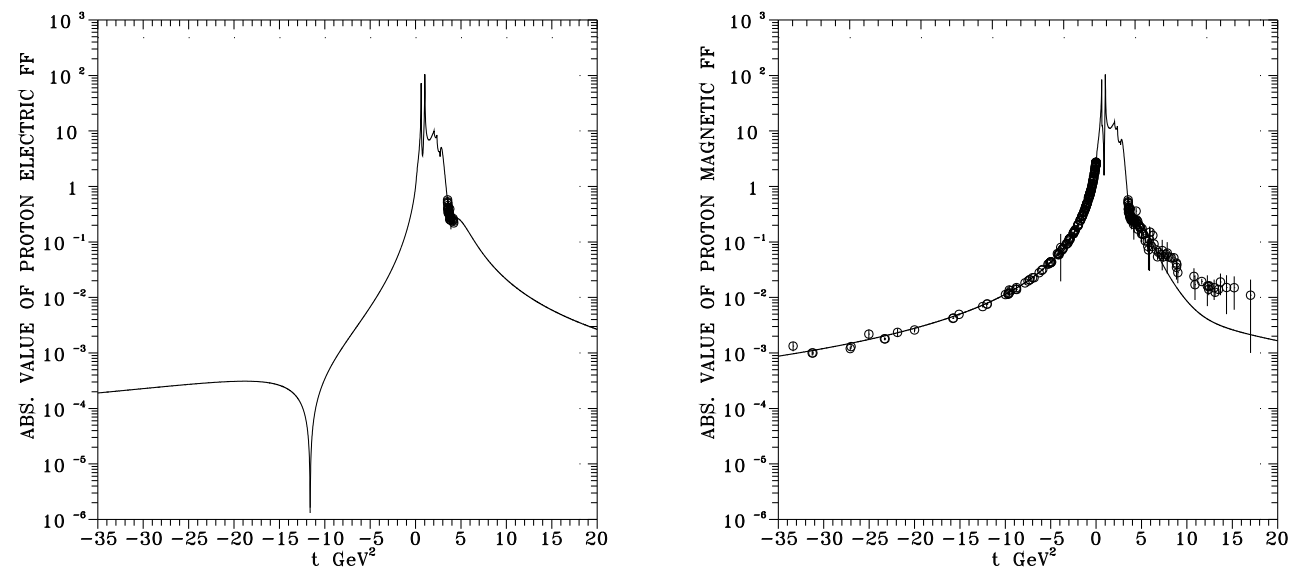

Figure 2: Prediction of proton electric and magnetic FFs behavior by $U \& A$ model respecting $S U(3)$ symmetry and OZI rule violation
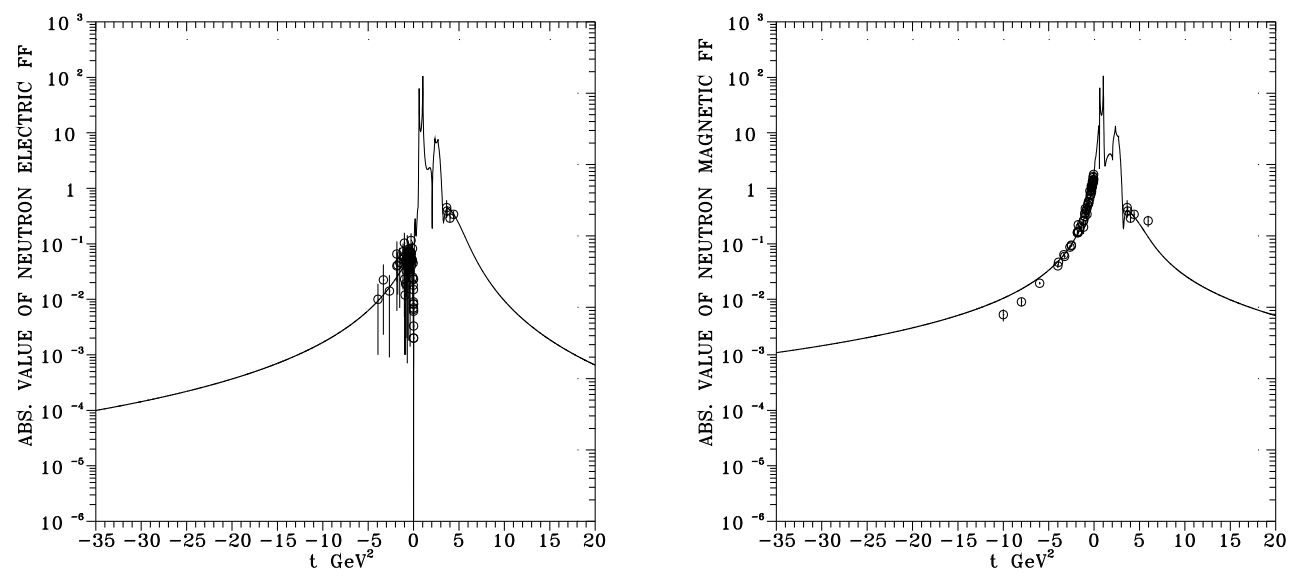

Figure 3: Prediction of neutron electric and magnetic FFs behavior by $U \& A$ model respecting $S U(3)$ symmetry and OZI rule violation 

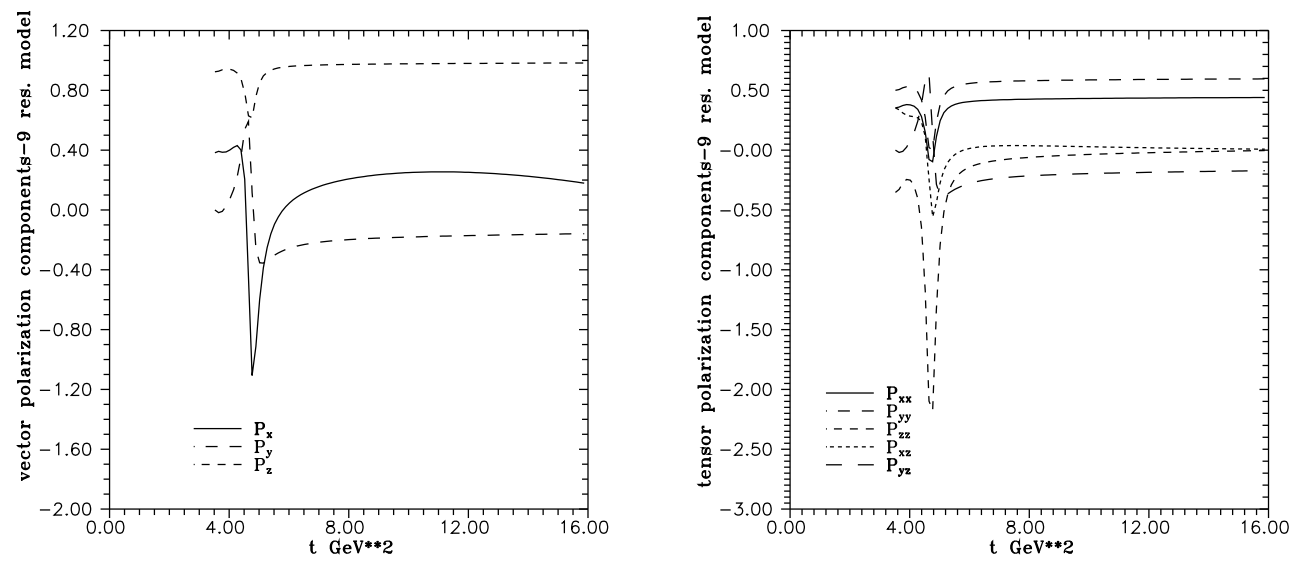

Figure 4: Vector and tensor polarization components of the final protons and antiprotons predicted by 9 resonance nucleon EM structure model.

$$
\begin{aligned}
& P_{y}=-\frac{\frac{1}{\sqrt{\tau}} \operatorname{Im}\left(G_{E p}(s) G_{M p}^{*}(s)\right) \sin 2 \vartheta}{\left|G_{E p}(s)\right|^{2} \sin ^{2} \vartheta / \tau+\left|G_{M p}(s)\right|^{2}\left(1+\cos ^{2} \vartheta\right)} \\
& P_{z}=\frac{2 \cos \theta\left|G_{M p}(s)\right|^{2}}{\left|G_{E p}(s)\right|^{2} \sin ^{2} \theta / \tau+\left|G_{M p}(s)\right|^{2}\left(1+\cos ^{2} \theta\right)}
\end{aligned}
$$

and tensor

$$
\begin{aligned}
& P_{x x}=\frac{\left|G_{M p}(s)\right|^{2} \cos ^{2} \vartheta-\frac{1}{\tau}\left|G_{E p}(s)\right|^{2} \sin ^{2} \vartheta}{\frac{1}{\tau}\left|G_{E p}(s)\right|^{2} \sin ^{2} \vartheta+\left|G_{M p}(s)\right|^{2}\left(1+\cos ^{2} \vartheta\right)} \\
& P_{y y}=\frac{\left|G_{M p}(s)\right|^{2}\left(1+\sin ^{2} \vartheta\right)-\frac{1}{\tau}\left|G_{E p}(s)\right|^{2} \sin ^{2} \vartheta}{\frac{1}{\tau}\left|G_{E p}(s)\right|^{2} \sin ^{2} \vartheta+\left|G_{M p}(s)\right|^{2}\left(1+\cos ^{2} \vartheta\right)} \\
& P_{z z}=\frac{\left|G_{M p}(s)\right|^{2} \sin ^{2} \vartheta-\frac{1}{\tau}\left|G_{E p}(s)\right|^{2} \cos ^{2} \vartheta}{\frac{1}{\tau}\left|G_{E p}(s)\right|^{2} \sin ^{2} \vartheta+\left|G_{M p}(s)\right|^{2}\left(1+\cos ^{2} \vartheta\right)} \\
& P_{x y}=P_{y x}=0 ; \\
& P_{x z}=P_{z x}=\frac{\frac{1}{\sqrt{\tau}} \operatorname{Re}\left[G_{M p}^{*}(s) G_{E p}(s)\right] \sin 2 \vartheta}{\frac{1}{\tau}\left|G_{E p}(s)\right|^{2} \sin ^{2} \vartheta+\left|G_{M p}(s)\right|^{2}\left(1+\cos ^{2} \vartheta\right)} \\
& P_{y z}=P_{z y}=-2 \frac{\frac{1}{\sqrt{\tau}} \operatorname{Im}\left[G_{M p}^{*}(s) G_{E p}(s)\right] \sin \vartheta}{\frac{1}{\tau}\left|G_{E p}(s)\right|^{2} \sin ^{2} \vartheta+\left|G_{M p}(s)\right|^{2}\left(1+\cos ^{2} \vartheta\right)}
\end{aligned}
$$

polarizations components of the final protons and antiprotons in the $e^{+} e^{-} \rightarrow p \bar{p}$ process, as they are presented in Fig. 4.

\section{4. $1 / 2^{+}$octet hyperons EM structure}

One can use the advanced $U \& A$ nucleon EM structure model for a description of the complete $1 / 2^{+}$octet hyperons EM structure, to be represented by the Sachs electric $G_{E}^{h}(t)$ and magnetic $G_{M}^{h}(t)$ FFs of hyperons. 
They can be decomposed into the flavor independent iso-scalar and iso-vector parts of the Dirac and Pauli FFs

$$
\begin{aligned}
& G_{E}^{\Lambda}(t)=F_{1 s}^{\Lambda}(t)+\frac{t}{4 m_{\Lambda}^{2}} F_{2 s}^{\Lambda}(t) \\
& G_{M}^{\Lambda}(t)=F_{1 s}^{\Lambda}(t)+F_{2 s}^{\Lambda}(t) \\
& G_{E}^{\Sigma^{0}}(t)=F_{1 s}^{\Sigma}(t)+\frac{t}{4 m_{\Sigma}^{2}} F_{2 s}^{\Sigma}(t) \\
& G_{M}^{\Sigma^{0}}(t)=F_{1 s}^{\Sigma}(t)+F_{2 s}^{\Sigma}(t) \\
& G_{E}^{\Sigma+, \Sigma^{-}}(t)=\left[F_{1 s}^{\Sigma}(t) \pm F_{1 v}^{\Sigma}(t)\right]+\frac{t}{4 m_{\Sigma}^{2}}\left[F_{2 s}^{\Sigma}(t) \pm F_{2 v}^{\Sigma}(t)\right] \\
& G_{M}^{\Sigma+, \Sigma^{-}}(t)=\left[F_{1 s}^{\Sigma}(t) \pm F_{1 v}^{\Sigma}(t)\right]+\left[F_{2 s}^{\Sigma}(t) \pm F_{2 v}^{\Sigma}(t)\right] \\
& G_{E}^{\Xi^{0}, \Xi^{-}}(t)=\left[F_{1 s}^{\Xi}(t) \pm F_{1 v}^{\Xi}(t)\right]+\frac{t}{4 m_{\Xi}^{2}}\left[F_{2 s}^{\Xi}(t) \pm F_{2 v}^{\Xi}(t)\right] \\
& G_{M}^{\Xi^{0}, \Xi^{-}}(t)=\left[F_{1 s}^{\Xi}(t) \pm F_{1 v}^{\Xi}(t)\right]+\left[F_{2 s}^{\Xi}(t) \pm F_{2 v}^{\Xi}(t)\right]
\end{aligned}
$$

Now, it is sufficient to substitute for the iso-scalar and iso-vector parts of the Dirac and Pauli FFs the expressions from nucleons, however, with the following change in the corresponding coupling constant ratios as free parameters of the model

$N \Rightarrow \Lambda, \Sigma^{0}, \Sigma^{ \pm}, \Xi^{0,-}$

In nucleons we have determined them in a fitting procedure of all existing data in space-like and time-like data simultaneously. In the case of hyperons a reliable data are missing.

But also in such case on finds the solution. The $S U(3)$ invariant Lagrangian

$$
\begin{array}{r}
\operatorname{Tr}\left(L_{V B \bar{B}}\right)=\frac{i}{\sqrt{2}} f^{F}\left[\bar{B}_{\beta}^{\alpha} \gamma_{\mu} B_{\gamma}^{\beta}-\bar{B}_{\gamma}^{\beta} \gamma_{\mu} B_{\beta}^{\alpha}\right]\left(V_{\mu}\right)_{\alpha}^{\gamma}+ \\
\frac{i}{\sqrt{2}} f^{D}\left[\bar{B}_{\gamma}^{\beta} \gamma_{\mu} B_{\beta}^{\alpha}+\bar{B}_{\gamma}^{\alpha} \gamma_{\mu} B_{\gamma}^{\beta}\right]\left(V_{\mu}\right)_{\alpha}^{\gamma}+ \\
\frac{i}{\sqrt{2}} f^{S} \bar{B}_{\beta}^{\alpha} \gamma_{\mu} B_{\alpha}^{\beta} \omega_{\mu}^{0}
\end{array}
$$

provides the relations between the vector-meson octet baryons coupling constants and the $S U(3)$ Lagrangian coupling constants $f^{F}, f^{D}$ and $f^{S}$. The latter can be found from the known nucleon coupling constants. However, in order to find all unknown coupling constats ratios in the model of hyperons, one has to know lepton decay widths of all considered trinity vector mesons. And they are known only for ground states of $\rho, \omega, \phi$.

We wait for measurements from Novosibirsk [4] and then one can cary out the whole program of a prediction of the hyperon EM FFs behavior.

The support of the Slovak Grant Agency for Sciences VEGA under Grant No.2/0009/10 and Grant No. 1/0158/13 is acknowledged.

\section{References}

[1] J.Beringer et al (Particle Data Group), Phys. Rev. D86 (2012) 010001.

[2] C.Adamuscin, S.Dubnicka, A.Z.Dubnickova, P.Weisenpacher, Prog.Part.Nucl.Phys. 55 (2005) 228.

[3] A.Z.Dubnickova, S.Dubnicka, M.Erdelyi, Prog.Part.Nucl.Phys 61 (2008) 162

[4] V.Sidorov, private communications 